\title{
MÉTODO DE MAPEAMENTO E REORGANIZAÇÃO DE PROCESSOS: SISTEMOGRAFIA
}

\section{METHOD OF MAPPING AND PROCESS REORGANIZATION: SYSTEMOGRAFY}

\author{
Fernando Ernesto Kintschner \\ Analista de Sistemas \\ Faculdade de Análise de Sistemas, Puc-Campinas \\ Rod. Dom Pedro I, KM 136 Parque das Universidades \\ Campinas - SP \\ (19) 3756-7162 - fek@ puc-campinas.edu.br \\ Ettore Bresciani Filho \\ Engenheiro Aeronáutico (Produção) \\ Faculdade de Análise de Sistemas, Puc-Campinas \\ Faculdade Engenharia Mecânica, Unicamp \\ Rod. Dom Pedro I, KM 136 Parque das Universidades \\ Campinas - SP \\ (19) 3756-7162 - brescia@ @lexxa.com.br
}

\begin{abstract}
Resumo
A partir da necessidade de se reduzir custos e aumentar a competitividade, as empresas iniciaram o processo de reorganização das áreas administrativas e de produção. A reorganização de processos envolve uma análise completa dos fluxos de trabalho e processos nas empresas. Porém, a maioria dos projetos de reorganização de processos falha. Neste contexto, da engenharia de sistemas, a partir do enfoque sistêmico, é apresentada a sistemografia para o mapeamento e reorganização dos processos.

Palavras-chave:Engenharia de sistemas, sistemas complexos, sistemografia.
\end{abstract}

Abstract

From the needs of decreasing costs and increasing competition, the companies initiate the process reorganization of alministration and production areas. The process reorganization implies a complete analyses of the workflow and process of the company. But, the most of the projects of process reorganization fails. In this context, the system engineering from the systemic approach, present sistemography to the mapping and process reorganization.

Key Words:systems engineering, complex systems, systemograph. 


\section{INTRODUÇÃO}

Em estudos realizados em empresas americanas, aproximadamente, $70 \%$ dos projetos de reorganização de processos falham (GRANT, 2002). Para que se atendam às expectativas das empresas, é fundamental a inserção de uma disciplina neste contexto: Engenharia de Sistemas.

A Engenharia de Sistemas envolve a aplicação de esforços para transformar uma necessidade do cliente em parâmetros de um sistema, através de um processo interativo de análise funcional, síntese, reorganização, definição, projeto, ensaio e avaliação. O termo sistema deve ser entendido como um conjunto de elementos que interagem para atingir um determinado fim, de acordo com um plano pré-determinado (BRESCIANI e D'OTTAVIANO, 2000).

O enfoque sistêmico procura disciplinar o bom-senso e a intuição através de um processo lógico e de uma análise formal do problema, procurando estudá-lo como um todo, preocupando-se com as interfaces entre suas diversas partes, enfatizando a necessidade de interação e avaliação permanente. (INPE, 1972, p. 18)

A Engenharia de Sistemas não é considerada uma área da engenharia, como a mecânica ou a elétrica, mas uma atividade que aplica um processo de desenvolvimento de sistema, da necessidade inicial identificada até o atendimento da mesma. (BLANCHARD, 1997, p. 35) E ela surgiu a partir da necessidade de se lidar com a complexidade de projetos de desenvolvimento de sistemas e com a aplicação de técnicas de modelagem e simulação para o desenvolvimento de processos que reorganizem os sistemas. Pode-se considerar que um dos maiores desafios de se 
projetar sistemas é encontrar, entre as muitas alternativas para o processo analisado, qual é a mais eficaz e eficiente.

Além disso, essa Engenharia está dividida em caracterização do problema e análise de sistemas. A caracterização do sistema cuida da criação, implementação e modificação de sistemas e, a análise de sistemas é responsável pela escolha entre várias alternativas possíveis de solução do problema. E também tem como principal requisito a análise dos processos envolvidos no domínio do problema, devendo ser identificados, mapeados e detalhados para que se tenha o efetivo entendimento do problema apresentado e para sua posterior resolução.

\section{CONCEITOS DE PROCESSO}

Definições sobre processo são encontradas em diversos ramos da ciência e sempre com significados semelhantes, embora sejam tratados em assuntos diferentes.

Para Harrington (1993, p.10), “o processo é definido como qualquer atividade que recebe uma entrada (input), agrega-lhe valor e gera uma saída (output) para um cliente interno ou externo, fazendo uso dos recursos da organização para gerar resultados concretos.” Já para Côrtes; Chiossi (2001, p.67), "um processo integra pessoas, ferramentas e métodos para executar uma sequiência de passos com o objetivo definido de transformar determinadas entradas em determinadas saídas".

Andersen (1999, p.03) entende processo como uma série lógica de transações que converte entrada para resultados ou saídas. Por outro lado, Gonçalves (2000a, p. 10) considera “processo mais do que a transformação de entradas em saídas, mas também o envolvimento de 
endpoints, feedback e repetibilidades". Já para Davenport (1994, p.25), "processo é uma ordenação específica das atividades no tempo e espaço, com começo e fim identificados".

Assim, um processo é composto de entradas, saídas, tempo, espaço, ordenação, objetivos e valores que resultam em uma estrutura para fornecer serviços e produtos aos clientes.E, sua importância nas empresas é confirmada através da constatação de que empresas industriais japonesas investem $70 \%$ de seus fundos de pesquisa e desenvolvimento em inovação de processos e têm resultados muito superiores ao de empresas americanas que investem a mesma proporção em desenvolvimento de produtos. (GONÇALVES, 2000b, p.17)

\section{REORGANIZAÇÃO DE PROCESSOS}

Após a onda de reengenharia aplicada nas empresas no início da década de 1990, em que havia mudanças radicais nos processos e nas estruturas empresariais, surge o conceito de reorganização dos processos de negócios, na qual as mudanças ocorrem de forma incremental e contínua com participação de todos os envolvidos. (KETTINGER; TENG, 2000)

A reorganização de processos envolve a análise e a definição de fluxos de trabalho e processos nas empresas, e cujo aumento da sua importância está associado ao movimento TQM (Total Quality Management), no qual os especialistas em qualidade focalizam a mudança incremental e gradual como sendo um fator para a melhoria desses processos (MALHOTRA, 1998). Neste contexto, a importância do paradigma da mudança radical na reorganização de processos é reduzida.

Pesquisas mostram que as reorganizações de processos nas empresas ocorrem a partir de mudanças radicais ou mudanças evolutivas (MEYERSON, 2001). As mudanças evolutivas, vistas isoladamente, não são perceptíveis, mas acumuladas, tendo-se o mesmo resultado das 
mudanças radicais, sendo que sua principal vantagem é que ocorrem menos conflitos dentro da empresa. A reorganização de processos deve ser realizada preferencialmente de forma evolutiva, porém, deve ser ressaltado que em algumas áreas não existe essa possibilidade, devido à natureza das atividades envolvidas, sendo necessária uma mudança radical.

Conforme Changchien; Shen (2002, p.347) e Kettinger et al (1997, p.55:80), as principais etapas da reorganização de processos são:

- $\quad$ análise dos processos atuais: verificar como a empresa trabalha atualmente e suas necessidades;

- $\quad$ análise de novas tecnologias: verificar novas tecnologias que possam ser utilizadas nos processos a serem alterados;

- $\quad$ definição da visão futura dos processos: projetar as alterações desejadas nos processos e;

- $\quad$ implantação da reorganização: colocar em prática os novos processos e verificar sua performance.

Durante a fase de reorganização de processos, alguns princípios adaptados de Hammer (2001, p.82:93) devem ser seguidos:

- cada processo deve ser projetado como uma unidade, devendo-se entendê-lo e reorganizá-lo do início ao fim;

- nenhuma atividade deve ser executada mais de uma vez, ou seja, não se deve tolerar duplicação e redundância de atividades e;

- o processo deve ser realizado por quem esteja mais bem preparado para fazê-lo, devendo-se verificar quem é a melhor pessoa ou até mesmo outra empresa para fazer a atividade. 


\section{TÉCNICAS DE MAPEAMENTO E REOGANIZAÇÃo DE PROCESSOS}

Segundo Kettinger et al (1997), há no mínimo setenta e duas técnicas usadas para acompanhar o mapeamento e a reorganização de processos, sendo que a maioria dessas técnicas foi desenvolvida para outros contextos e, foram adaptadas a este trabalho, como por exemplo, as técnicas de qualidade e as técnicas de modelagem de banco de dados.

As principais técnicas de mapeamento de processos são os mapas e diagramas que permitem ao modelador discutir e validar modelos de processos com os usuários. Essas técnicas levam à aplicação da heurística, onde a experiência dos modeladores e seus conhecimentos do negócio modelado influenciam a criação dos diagramas e mapas (PHALP; MARTIN,2000).

Outras técnicas utilizadas como complemento para a reorganização de processos segundo Kettinger et al. (1997) são as técnicas criadas para o controle de qualidade, como o diagrama Ishikawa (espinha de peixe), histograma, QFD (Quality Function Deployment) e o diagrama de Pareto entre outros. Pode-se citar como técnicas também utilizadas: rede de Petri, redes de controle de informação, diagrama de regras de atividades e análise de campo de força.

Porém, nota-se que, nestas técnicas de mapeamento de processos, sempre é necessário se usar mais de uma em conjunto para se ter um resultado eficaz. Isto torna o mapeamento demorado, quando grande e complicado. Assim, o recomendado é que se utilize uma única técnica de mapeamento que consiga englobar todos os aspectos do processo e também tenha facilidade de uso igual ou melhor às descritas anteriormente.

Há alguns modelos que tomam como base essa recomendação como Aalst (2000) com as redes de Pedri, Phalp e Martin (2000) com o diagrama de regras de atividades, Johannesson e 
Perjons (2001) com a linguagem de modelagem de negócios (BML) e o IDEF criado pelo governo americano. (NUMA, 2002) Neste trabalho é introduzido o modelo denominado sistemografia devido a sua facilidade para mapeamento de processos simples e complexos.

\section{SISTEMOGRAFIA E SISTEMÓGRAFO}

A partir da Engenharia de Sistemas, que envolve a aplicação de esforços para a análise funcional e a reorganização de processos e, utilizando-se dos fundamentos da Teoria do Sistema Geral (Bertalanffy,1968), Le Moigne (1990) descreveu os conceitos básicos sobre o método chamado Sistemografia. Posteriormente, Bresciani (2002) descreveu operacionalmente esse modelo.

A sistêmica é uma teoria de modelagem de sistemas complexos que decorre dos estudos desenvolvidos principalmente a partir da Teoria do Sistema Geral. Nela se substitui o enfoque analítico, em que a principal questão é “do que isto é feito?" pelo enfoque sistêmico em que a questão é “o que isto faz?”.

Segundo Bresciani; D’Ottaviano (2000, p.283:300), o enfoque sistêmico tem alguns preceitos básicos:

- a existência do sistema, com uma estrutura subjacente, constituída por um conjunto de elementos e pelas relações entre esses elementos, e com uma funcionalidade;

- a caracterização dos elementos internos, externos e de fronteira do sistema;

- a existência das propriedades de sinergia, globalidade e novidade;

- a presença de um sujeito observador do sistema, de natureza complexa e com a possibilidade de ser externo, interno ou de fronteira; 
- a possibilidade de o sistema receber do exterior (meio-ambiente), transformar internamente e transmitir para o exterior (meio-ambiente), através de uma fronteira (arbitrária), energia, matéria e informação;

- a identificação de relações de distintos graus de complexidade entre os elementos do sistema;

- a identificação de uma propriedade do sistema, caracterizada por sua estrutura e pelo seu funcionamento, denominada organização, que conduz o comportamento do referido sistema;

- a existência das propriedades teleológicas (nas quais o objeto é interpretado pelo seu comportamento) e de equifinalidade do sistema;

- a criação, no sistema, de condições restritivas e de perturbação, de características determinadas (certas) e indeterminadas (incertas) devido à interação com o meioambiente através de sua fronteira (arbitrária);

- a necessidade da existência de um campo de influência (ou de forças) para provocar um fluxo de atividades;

- a possibilidade de manutenção de equilíbrio estrutural e funcional, ou seja, da manutenção do estado do sistema nas relações com o meio-ambiente, através do mecanismo da regulagem;

- a possibilidade de mudança de estado com a emergência de novo estado, que caracteriza a criação ou a evolução, através do mecanismo de adaptação estrutural e funcional; 
- a possibilidade da presença do fenômeno de auto-organização, decorrente da interação das atividades predeterminadas do sistema com as atividades autônomas e espontâneas dos elementos do sistema em um processo recorrente e;

- a possibilidade de transformações através de processos criativos, que podem ser decorrentes do fenômeno de auto-organização.

A sistêmica utiliza-se de uma técnica de construção de modelos complexos chamada sistemografia.Então sistemografar é construir um modelo de um fenômeno percebido como complexo, sendo uma representação do sistema através de uma exemplificação idealizada da realidade, com o propósito de explicar as relações essenciais envolvidas. Assim, a sistemografia constrói um fluxograma de um processo sistêmico com a denominação de sistemógrafo.

A sistemografia é a representação de atividades, identificados por um modelador, por meio de um sistema, utilizando-se para tanto da Teoria do Sistema Geral que tem como objetivo a compreensão do comportamento dos sistemas complexos em relação aos seus diversos componentes e o meio-ambiente. É uma teoria criada na tentativa de modelagem de sistemas abertos (que interagem com o meio-ambiente), em contrapartida ao tradicional método analítico (tratamento de sistemas fechados). (SCHODERBEK,1990, p 5-32) E, diferentes modeladores podem construir diferentes sistemógrafos.

Para a criação do sistemógrafo, a sistemografia classifica as atividades que estão dentro da fronteira do projeto por: família (objetos processados ou objetos processadores), por categoria (operacional, informacional e decisional), por tipo (forma, espaço e tempo) e por nível de complexidade $\left(1^{\circ}\right.$ a $\left.9^{\circ}\right)$. 


\section{a) Classificação por família}

A atividade é representada pelo que ele faz (processador) ou pelo resultado de algo que nele foi feito (processado). A atividade pode ser considerada como uma caixa-preta, com a sua constituição interna não definida e, portanto, não necessária para sua compreensão.

\section{b) Classificação por categoria}

As atividades constituem sistemas que, por sua vez, podem ser classificados em três categorias: sistema operacional, sistema informacional e sistema decisional. O sistema operacional é constituído pelas atividades desenvolvidas; o sistema informacional é constituído da utilização de informações para se desenvolver as atividades e o sistema decisional é constituído pelo conjunto de decisões tomadas a partir das informações para realização das atividades.

\section{c) Classificação por tipo}

A atividade deve ser classificada pelos tipos: tempo, espaço e forma. A definição do tipo da atividade deve ser feita a partir das alterações no comportamento do objeto que está sendo referenciado na atividade em relação aos referenciais tempo (se o tempo gasto para executar a atividade foi relevante), espaço (se a atividade ocasionou o deslocamento relevante de algo) e forma (se a atividade alterou ou converteu algo relevante), sendo que cada atividade pode ser classificada por um ou mais tipos.

\section{d) Classificação por nível de complexidade}

Conforme Kintschner (2003, p.38), a atividade deve ser classificada em um dos nove níveis progressivos de complexidade:

$1^{\text {o }}$ - Atividade passiva: a atividade é inerte e não exerce qualquer processamento. Exemplo: alguma área da empresa que tem como atividade receber um documento e, simplesmente, repassá-lo para outra sem qualquer verificação ou processamento. 
$2^{\circ}$ - Atividade ativa: a atividade processa, realiza e exterioriza um comportamento. Exemplo: todas as atividades que tenham algum tipo de processamento.

$3^{\circ}$ - Atividade regulada: a atividade também processa, realiza e exterioriza um comportamento, porém, manifesta uma efetiva regularidade em sua atividade. Exemplo: a atividade de retirada de amostra de matéria-prima recebida para verificação da qualidade.

4- Atividade informada: a atividade também processa, realiza e exterioriza um comportamento de forma regular, porém, utilizando informação de forma relevante. Exemplo: a atividade de dar baixa nos materiais do estoque, de acordo com a requisição do usuário e disponibilidade no estoque.

$5^{\text {o- }}$ Atividade com decisão: a atividade tem capacidade de tomar decisão com base em uma informação que provoca uma ação pré-definida e conhecida. Exemplo: o processo de rejeitar o recebimento de um material devido ao problema na qualidade.

$6^{\circ}$ - Atividade com memória: a atividade além de tomar decisão apóia-se em um processo de memorização; a representação é feita com processador decisional. Exemplo: consulta a área de compras de materiais para verificar procedimentos a serem tomados em relação a um fornecedor.

$7^{\circ}$ - Atividade com coordenação: a atividade articula-se segundo três subsistemas agregados e fundamentais: decisional, informacional e operacional; o processador decisional deve ter a capacidade de coordenação que implica na capacidade relacional, ou seja, número de outros processadores com os quais se conecta, e a capacidade de tratamento de informação. Exemplo: atividades de tomada de decisões rotineiras tomadas pela direção de uma empresa.

$8^{\text {o- }}$ Atividade com inovação: a atividade tem a capacidade de inovação (imaginação, seleção, concepção, criação e invenção), de gerar informação simbólica, de aprendizagem, de 
inteligência, e de auto-organizar-se. Exemplo: atividades da área de marketing da empresa com capacidade de inovar, através de campanhas, os produtos da empresa.

9 ${ }^{\text {- }}$ Atividade com autofinalização: a atividade passa a ter no seu sistema de coordenação a capacidade de gerar os seus próprios objetivos e de ter consciência da sua existência e identidade. Essa atividade tem, ainda, a capacidade de definir diagnóstico e manutenção. Exemplo: atividade de tomada de decisões autônomas de estabelecimento de políticas, realizada pela direção de uma empresa.(ver quadro 5.1)

\section{ETAPAS PARA MAPEAMENTO E REORGANIZAÇÃO DE PROCESSOS}

Pode-se sugerir as seguintes etapas para o mapeamento e reorganização de processos adaptados de Bresciani (2002) e Kintschner (2003, p.40):

\section{QUADRO 5.1 - EXEMPLO DE CLASSIFICAÇÃO DE SISTEMÓGRAFO}

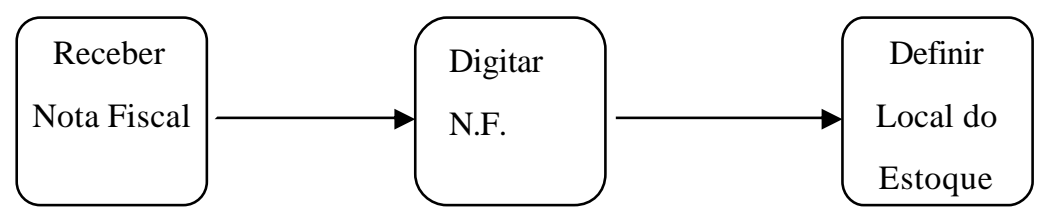

Considere um sistema de recebimento de materiais: a nota fiscal de material recebida na empresa (atividade 1) é enviada para digitação (atividade 2) e, logo em seguida, é definido o local de armazenagem e criado o documento de identificação do material (atividade 3). Classificando-se os objetos por tipo e complexidade:

\section{Atividade}

1 - Recebe nota fiscal

2 - Digitar nota fiscal

3 - Definir local do estoque

$\begin{array}{lc}\text { Referencial alterado } & \text { Complexidade } \\ \text { espaço/tempo } & 2^{\circ} \\ \text { forma/tempo } & 4^{\mathrm{o}} \\ \text { espaço/tempo/forma } & 4^{\mathrm{o}}\end{array}$

A função da atividade 1 é de transporte; há um deslocamento do documento, alterando o espaço na atividade processada (nota fiscal) e sua complexidade é do $2^{\circ}$ nível, pois, apesar de manter uma certa regularidade, não possui um meio de controle automático, podendo sofrer alterações de comportamento provocadas por algum fator externo como, por exemplo, um aumento nas compras. 
forma.

A função da atividade 2 é a transformação (digitação) da nota fiscal, alterando sua

A função da atividade 3 é a de transformação e transporte, pois há um novo documento para estocagem do material e este é deslocado para o local definido, alterando a forma e espaço.

As três atividades alteram o tempo, pois cada uma utiliza um determinando tempo na execução da sua tarefa. E, as atividades 2 e 3 são de $4^{\circ}$ nível, pois têm a capacidade principal de processar informações .

$1^{\mathrm{a}}$ Definir a fronteira do sistema a ser modelado, fixando os processadores de fronteira, ou seja, aqueles responsáveis pelas entradas e saídas do sistema.

$2^{\text {a }}$ Construir o sistemógrafo operacional do sistema, dispondo em um fluxograma as diferentes atividades operacionais.

$3^{\text {a }}$ Construir o sistemógrafo informacional do sistema, dispondo em um fluxograma as diferentes atividades que utilizam o processamento de informação, sendo que essas informações tipicamente processadas são de fornecedores, de pedidos, estoque, produção, entre outros.

$4^{\mathrm{a}}$ Construir o sistemógrafo decisional do sistema de produção, dispondo em um fluxograma as diferentes atividades que tiveram processo de decisão e representando cada uma segundo os mesmos critérios adotados para os outros dois sistemas (operacional e informacional). É importante notar que a tomada de decisão apóia-se na informação disponível e afeta a operação.

$5^{\text {a }}$ Classificar todos os processadores do sistemógrafo em categorias, tipos e níveis e construir uma tabela comparativa. Esse procedimento permite o processo de busca da reorganização, flexibilidade e agilidade do sistema.

$6^{\text {a }}$ Relacionar os problemas em uma ordem de prioridade, adotando-se critérios qualitativos e quantitativos, identificar e encontrar soluções para os problemas que levem às modificações operacionais, informacionais, decisionais e organizacionais (funcionais e estruturais) e 
ao estabelecimento de estratégias, visando a acompanhar e a controlar o processo de evolução do sistema.

$7^{\text {a }}$ Reduzir, aglutinar ou eliminar atividades que envolvam pelo menos um dos itens abaixo:

- $\quad$ níveis baixos de complexidade (menor que três);

- $\quad$ somente tipos espaço e tempo, isto é, se a atividade não alterou ou converteu o objeto e;

- $\quad$ somente categoria operacional.

$8^{\mathrm{a}}$ Propor a solução dos problemas na forma de recomendações estratégicas e de procedimentos e, em uma fase posterior, implantar, acompanhar e aprimorar as mudanças tecnológicas e administrativas adotadas.

\section{CONCLUSÕES}

A simplicidade da modelagem é constatada a partir do seu núcleo central, que utiliza a sistemografia para identificar as atividades, sendo que a classificação das atividades em categorias, tipos e níveis e a construção de uma tabela comparativa de atividades torna o processo de busca da reorganização flexível e ágil.

Portanto, com a utilização da sistemografia é possível identificar atividades redundantes e atividades que podem ser eliminadas ou aglutinadas por meio de suas descrições e classificações. 


\section{REFERÊNCIAS BIBLIOGRÀFICAS}

AALST, W. Loosely coupled interorganizational workflows: modeling and analyzing workflows crossing organizational boundaries. Information \& Management, Breukelen, v.37, p.67-75, 2000.

ANDERSEN, B. Business process improvement toolbox. Milwaukee,Wisc.: ASQ, 1999.

BERTALANFFY, L.V. Teoria Geral dos Sistemas. Petrópolis: Editora Vozes, 1968.

BLANCHARD, B.S. System Engineering Management. New York: J.Wiley, 1997.

BRESCIANI, FILHO Ettore; D'OTTAVIANO, Itala Maria Loffredo. Conceitos básicos de sistêmica. Auto-Organização: Estudos Interdisciplinares, Campinas: CLE / Unicamp, v.2, p.283306, 2000.

BRESCIANI, FILHO, Ettore. Método de estudo de sistema -sistemografia. Revista do Instituto de Informática da Puc-Campinas. Campinas. v.1, n.1, 2001. Disponível em <www.ii.puccampinas.br/revistaeletronica>. Acesso em 20/08/2002.

CHANGCHIEN, S.; SHEN,H. Supply chain reengineering using a core process analysis matrix and object-oriented simulation. Information \& Management, Breukelen, v.39, p.345-358, 2002.

CÔRTES, Mario Lúcio, CHIOSSI, Thelma C.S., Modelos de qualidade de software, Campinas: Editora da Unicamp, 2001.

DAVENPORT, Thomas. Reengenharia de processos. Rio de Janeiro: Editora Campus, 1994.

GONÇALVES, José E.L. As empresas são grandes coleções de processos. R.A.E. - Revista de Administração de Empresas - FGV, São Paulo, v.40, n.1, pp.6-19, Jan/Mar 2000a.

Processos, que processos? R.A.E. - Revista de Administração de Empresas - FGV, São Paulo, v.40, n.1, pp.8-19, Out/Dez 2000b.

GRANT, Delvin. A winder view of business process reengineering. Communications of the ACM, New York, v.45, n.2, 2002.

HAMMER, Michael. The super efficient company. Harvard Business Review, Boulder, v.79, n.8, p.82-93, 2001.

HARRINGTON, H.J. Aperfeiçoando processos empresariais. São Paulo: Makron Books, 1993.

INSTITUTO NACIONAL DE PESQUISAS ESPACIAIS (Brasil). Engenharia de sistemas: planejamento e controle de projetos. Petrópolis: Editora Vozes, 1972, 307p. 
JOHANNSESSON, P.; PERJONS, E.. Design principles for process modeling in enterprise application integration. Information Systems, v. 26, p.165-184, 2001.

KETTINGER, W.; TENG, J.; GUSHA, S. Business process change: a study of methodologies, techniques, and tools. MIS Quarterly, Minneapolis, v.21, n.1, p.55-80, 1997.

KETTINGER, W.; TENG, J. Business process change in the $21^{\text {st }}$. century. Bussiness \& Economic Review, v.46, n. 2, p.14-19. 2000.

KINTSCHNER, Fernando E. Método de Modelagem de Processos para Apoio ao Desenvolvimento de Software. 2003. 161 f. Tese (Doutorado em Engenharia Mecânica) Faculdade de Engenharia Mecânica, Universidade Estadual de Campinas, Campinas,2003.

LE MOINGE, La modélisation des systèmes complexes. Paris: Editora Dunod, 1990.

MALHOTRA, Yogesh. Business process: Overview. IEEE Engineering Management Review, v.26, n.3, Fall, 1998.

MEYERSON, Debra E. Radical change, the quiet way. Harvard Business Review, Boulder. v.79, n.9, p.92-104, 2001.

NUMA, Núcleo de Manufatura Avançada -Universidade de São Paulo. Escola de Engenharia de São Carlos. Disponível em 〈www.numa.org.br/transmeth/ferramentas/ffmapeam.htm>. Acesso em: em 20/05/2002.

PHALP, Keith; MARTIN, Shepperd. Quantitative analysis of static models of process. The Journal of Systems and Software, v.52, pp.105-112, 2000.

SHODERBEK, Shoderbek. Management System - Conceptual Considerations. BPI IRWIN, 1990, p.5-32. 